\title{
Impregnación en polines de híbridos de álamo tomando como referencia polines impregnados de pino
}

\author{
Preservative treatment in poles of poplar hybrids taking radiata pine poles as a reference
}

\author{
MARCIA VASQUEZ S., FABIAN PEREDO D. \\ Departamento de Industrias Forestales, Facultad de Ciencias Forestales \\ Universidad de Talca, Casilla 721, Talca, Chile \\ Email:mvasquez@utalca.cl
}

\begin{abstract}
SUMMARY
The behavior difference of poplar utility poles after impregnation was evaluated and the physical and mechanical properties of impregnated wood were characterized using wood coming from the top useful part of the log from three commercial hybrids of poplar, such as: I-214, I-488 and I-63/51. Radiata pine utility poles were used as a control. They were impregnated and tested under the same conditions as the poplar hybrids to determine their physical and mechanical properties. Thirty utility poles of poplar and ten of pinus and were impregnated with CCA using the Bethell method. It was found that the hybrid I-488 showed better impregnation behavior than the pine and the hybrid I-214 showed better mechanical properties than the pine. It was observed that the hybrid I-63/51 showed some problems in impregnation and similar mechanical behavior to that of the pine. Based on this study it could be inferred that impregnated utility poles of hybrids I-214 and I-488 could be utilized the same as impregnated pine utility poles.
\end{abstract}

Key words: poplar, utility poles, impregnation, physical and mechanical properties.

\section{RESUMEN}

Se evaluó la diferencia en el comportamiento de polines de álamo frente a la impregnación y se caracterizaron las propiedades físicas y mecánicas de la madera impregnada proveniente de la última troza utilizable del fuste de tres híbridos de álamo de interés comercial, como son: I-214, I-488 e I-63/51. Se utilizaron como testigo polines de Pinus radiata D. Don, los que fueron impregnados y ensayados bajo las mismas condiciones que los híbridos de álamo para determinar sus propiedades físicas y mecánicas. Para ello se utilizaron 30 polines de los híbridos de álamo y 10 polines de pino, los cuales fueron impregnados con sales de cobre, cromo y arsénico (CCA) utilizando el método Bethell. Se comprobó que el híbrido I-488 presenta mejor comportamiento frente a la impregnación que el pino y que el híbrido I-214 presenta mejor comportamiento mecánico que el pino. El híbrido I-63/51 presentó problemas de impregnación y similar comportamiento mecánico que el pino. De lo anterior se puede inferir que los polines impregnados de los híbridos I-214 e I-488 podrían ser empleados como polines impregnados al igual que el pino.

Palabras clave: álamo, polines, impregnación, propiedades físicas y mecánicas. 


\section{INTRODUCCION}

La VII Región del Maule concentra a nivel país la mayor cantidad de plantaciones comerciales de álamo. De acuerdo a cifras reportadas por el INIA existen 15.000 hectáreas plantadas, siendo 7.500 hectáreas de bosques productivos (1, 2). A nivel país se reportan alrededor de 20.000 hectáreas de plantación ocupando sólo un $0,2 \%$ de la superficie plantada (3). Además, el rendimiento en cosecha de plantaciones de álamo es bajo, porque un tercio del fuste queda en el bosque debido a su bajo diámetro y abundantes ramas que no lo permiten ser utilizable industrialmente. Es decir, no es mucho el recurso y además no se usa integralmente. Sin embargo, el corto período de rotación (de 12 a 14 años) y buenas propiedades de la madera de álamo como: liviana, blanda, dúctil, fácil de trabajar (debobinar, aserrar, teñir, etc), justifican su gran extensión entre las Regiones IV a la XI (4). Las plantaciones de álamo están conformadas por híbridos que permiten obtener madera que varía en su color desde blanco-amarillento hasta café pálido, presentando una zona interna de color más oscuro, denominada falso duramen (5). Dentro de los híbridos comerciales más utilizados industrialmente en Chile tenemos: Populus x euroamericana cv I-214, cv I-488 y cv I-63/51. De acuerdo a algunas evaluaciones tecnológicas de la madera, el álamo es una especie poco durable; según NCh 789 Of 87 (6) su vida útil es inferior a 5 años, pero a su vez la madera es permeable y fácil de impregnar posibilitándola para usos constructivos $(7,8,9,10,11,12)$.

En base a lo anterior, se propuso un estudio para evaluar el comportamiento de polines provenientes de distintos híbridos de álamo frente al impregnado y para determinar las propiedades físico-mecánicas de la madera impregnada de álamo proveniente de la última troza no utilizable tomando como referencia el comportamiento y las propiedades de polines impregnados de pino (13).

\section{MATERIAL Y METODOS}

Se utilizaron un total de 30 polines distribuidos en igual proporción entre los híbridos I-214, I-488 e I-63/5, los que se obtuvieron de una faena llama- da de recuperación de la cosecha desde una plantación intensiva en manejo de 12 años de edad, ubicada en la zona de Retiro, VII Región. Las dimensiones de los polines fueron de 3 a 4 pulgadas de diámetro y 2,44 metros de longitud. Un número similar de 10 polines de pino y de iguales dimensiones fueron utilizados como testigo en el estudio.

Los polines de álamo se sometieron a un cilindrado y se dispusieron en un castillo triangular para su secado al aire. Un período total de dos meses fue necesario para bajar la humedad de la madera a la humedad de equilibrio del ambiente durante el período estival 2003. La impregnación se realizó en una planta piloto de $0,2 \mathrm{~m}^{3}$ de capacidad ubicada en el Centro Regional de Tecnología e Industria de la Madera (CERTIM) de la Facultad de Ciencias Forestales de la Universidad de Talca en Talca. Se utilizó el método Bethell con una solución al 1,3\% en óxidos de la sal preservante CCA tipo C.

Se realizaron cinco cargas mixtas de impregnación, conformada por dos polines de cada híbrido y dos polines de pino, siguiendo el procedimiento que se muestra en la figura 1.

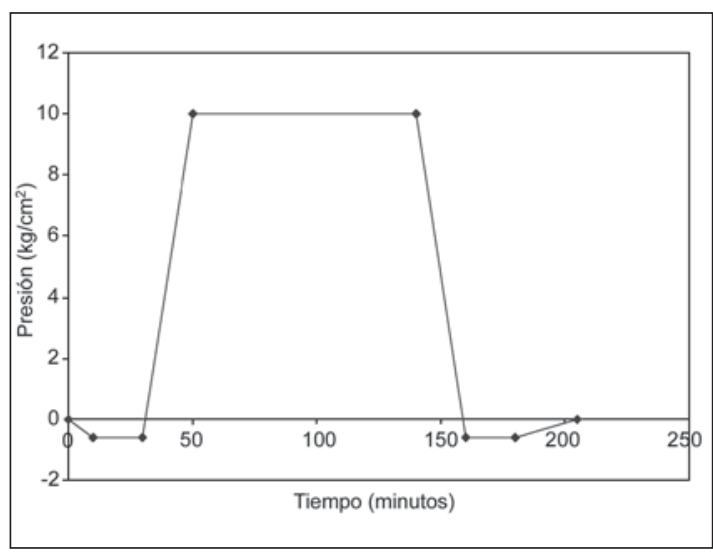

Figura 1: Condiciones del proceso de impregnado Bethell para los polines de los híbridos de álamo y polines de pino.

Bethell impregnation process conditions for hybrids of poplars poles and pinus poles.

Previo al impregnado se caracterizó físicamente la madera, determinando su contenido de humedad y densidad según NCh 176/1 Of. 84 (14) y NCh 176/2 Of. 86 (15). Posterior al impregnado se evaluó la eficacia del tratamiento mediante el cumplimiento de los siguientes parámetros: $6 \mathrm{~kg}$ óx. $/ \mathrm{m}^{3}$ de retención y penetración total - regular 
(TR) del preservante en la madera. La retención fue determinada a través de fluorescencia de rayos X usando el equipo ASOMA y la penetración a través de un ensayo colorimétrico usando reactivo de cromo asurol. La penetración del preservante debe ser regular o uniforme, siendo esta una medida cualitativa que es llevada a un valor cuantitativo, como es el porcentaje en que aparece distribuido el preservante en la sección de madera muestreada. La NCh 819 Of 96 (16) establece los requerimientos de la madera impregnada para pino según condiciones de riesgo. Estos requisitos fueron los adoptados para la madera impregnada de álamo, debido a la similitud de las maderas de baja densidad y poca durabilidad. Además se procedió a determinar las propiedades mecánicas de los polines impregnados de los distintos híbridos de álamo y la madera de los polines impregnados de pino, en probetas acondicionadas a un contenido de humedad de $12 \%$, según procedimiento señalado en la NCh 968 Of 86 (17).

Para realizar los ensayos mecánicos se seleccionaron cinco polines impregnados por especie, procedentes de cualquiera de las cinco cargas de impregnación. El criterio utilizado en la selección fue tomar los polines más homogéneos en sus retenciones, alrededor de $6 \mathrm{~kg}$ de óxidos por metro cúbico, valor de retención mínimo exigido por norma (16). De esta manera se reduce el efecto tratamiento en la respuesta de la madera frente a las diferentes solicitaciones.

Ensayos de compresión paralela y flexión estática se realizaron en probetas de pequeña dimensión siguiendo el método secundario estable- cido en las NCh 973 Of 85 (18) y NCh 987 Of 85 (19), respectivamente. Las probetas se ensayaron en la máquina universal marca Shenk Trebel de propiedad del Laboratorio de Resistencia de Materiales de la Facultad de Ingeniería de la Universidad de Talca en Curicó.

Con los datos obtenidos se procedió a realizar un estudio de comparación de medias y análisis múltiple con el software Stat Graphic.

\section{RESULTADOS Y DISCUSION}

El contenido de humedad y densidad básica de la madera y el comportamiento frente a la impregnación se muestran en el cuadro 1 .

La densidad de la madera de pino es más baja a la señalada en referencias, de 420 a $450 \mathrm{~kg} / \mathrm{m}^{3}$ $(20,21)$, propiedad determinada a la altura del pecho, igual a 1,3 m en árboles adultos. El valor más bajo de densidad podría atribuirse a la localización de la muestra, pudiendo provenir de la última troza no aserrable (diámetro del fuste inferior a $10 \mathrm{~cm}$ obtenido de una zona del árbol superior a los 20 metros de altura), o a polines que se generan producto de una actividad de manejo del bosque como el raleo en árboles jóvenes. Además, la madera de Pinus radiata posee una alta variabilidad de la densidad con respecto a la altura, habiendo estudiado esta condición varios autores como Burdon y Harris (22), Harris (23), Bamber y Burley (24), Wilkes (25), Ringo y Klem (26), Zobel y Van Buijtenen (27) y Cown et al. (28), entre otros.

\section{CUADRO 1}

Características de los polines impregnados con CCA. Valor promedio y desviación estándar (entre paréntesis).

Characteristics of utility poles treated with CCA. Average value and standard deviation (in parenthesis)

\begin{tabular}{|llccc|}
\hline Especie & $\begin{array}{c}\text { Contenido de } \\
\text { humedad }(\%)\end{array}$ & $\begin{array}{c}\text { Densidad } \\
\left(\mathrm{kg} / \mathrm{m}^{3}\right)\end{array}$ & $\begin{array}{c}\text { Retención } \\
(\mathrm{kg} \text { óxido/m }\end{array}$ & $\begin{array}{c}\text { Penetración } \\
(\%)\end{array}$ \\
\hline $\mathrm{I}-214$ & $12,2(0,33)$ & $419(23,3)$ & $5,98(2,16)$ & $70 \mathrm{TR}$ \\
$\mathrm{I}-488$ & $12,0(0,34)$ & $317(25,7)$ & $6,31(0,79)$ & $100 \mathrm{TR}$ \\
$\mathrm{I}-63 / 51$ & $12,0(0,19)$ & $380(22,0)$ & $5,38(1,22)$ & $60 \mathrm{TR}$ \\
Pino & $12,9(0,58)$ & $343(18,4)$ & $7,77(1,35)$ & $80 \mathrm{TR}$ \\
\hline
\end{tabular}


Los resultados de densidad básica de la madera de los híbridos en estudio se contradicen con algunos valores de densidad reportados por Véliz y Sepúlveda (29, 30). Ellos encontraron que la especie I-488 posee una densidad superior a la especie I-63/51. Los valores de densidad determinados fueron de $344 \mathrm{~kg} / \mathrm{m}^{3}$ y $323 \mathrm{~kg} / \mathrm{m}^{3}$ para el I-488 y de $296 \mathrm{~kg} / \mathrm{m}^{3}$ y $326 \mathrm{~kg} / \mathrm{m}^{3}$ para I-63/51. Las diferencias se explican principalmente por la zona desde donde fueron obtenidas las muestras para su determinación, siendo en este estudio obtenidas de la ultima troza del árbol, mientras que en los estudios señalados corresponden a la primera troza del árbol a la altura del pecho. En base a lo anterior se podría inferir que madera de álamo podría presentar un patrón distinto de variación de densidad en función de la altura e híbrido, como lo ha señalado el investigador suizo Lenz citado por FAO (31)

Bajo las condiciones de impregnación establecidas en el punto anterior, se puede observar que la madera impregnada del híbrido I-488 es la única que cumple con los requerimientos de retención y penetración, establecidos por la NCh 819 Of 96 (16). Contrario a este comportamiento, se puede observar que el híbrido que presenta mayor dificultad en la penetración de la solución es el I-63/51. La homogeneidad del tratamiento se mide a través de la desviación estándar del valor de retención, resultando más homogéneo el híbrido I-488 con una desviación estándar de 0,79 y el más heterogéneo el híbrido I-214, con una desviación estándar de 2,16. Las diferencias son atribuibles a las características anatómicas de la especie, ya que las especies corresponden al mismo sitio y manejo, y el tratamiento fue constante para todas ellas.
Con el fin de evaluar la significancia estadística de las diferencias entre medias observadas en el cuadro 1, se realizó el test estadístico de Dunnet con un nivel de confianza del $95 \%$ y 36 grados de libertad, considerando las siguientes hipótesis:

$$
\begin{aligned}
& \text { Ho: } \mu_{\mathrm{c}}=\mu_{\mathrm{ti}} \\
& \text { Ha: } \mu_{\mathrm{c}} \neq \mu_{\mathrm{ti}}
\end{aligned}
$$

Donde $\mathrm{i}=1=\mathrm{I}-214,2=\mathrm{I}-488$ y $3=\mathrm{I}-63 / 51$

El cuadro 2 muestra los resultados del análisis estadístico donde se evalúa la igualdad o diferencias entre las medias de la madera de pino impregnada y las medias del tratamiento de impregnación en los distintos híbridos de álamo.

Al analizar los resultados obtenidos del test se puede observar que los híbridos I-214 e I-488 no presentan diferencias estadísticamente significativas con respecto al pino. El híbrido I-63/51 presenta diferencias estadísticamente significativas con respecto al pino impregnado, siendo necesario hacer algún tipo de modificación al proceso para impregnar satisfactoriamente este híbrido. Es importante destacar que la madera de uso agrícola exige una retención mayor o igual a $6 \mathrm{~kg}$ de óxidos por metro cúbico, por lo que madera impregnada del híbrido I-63/51 bajo estas condiciones estaría en una situación de riesgo de degradación.

Los resultados sobre ensayos mecánicos de polines impregnados a distintas cargas de impregnante para las especies se aprecian en el cuadro 3.

Los cuadros síntesis 4 y 5 muestran en forma clara que existen diferencias estadísticamente sig-

\section{CUADRO 2}

Comparación entre valores medios de retención de los híbridos de álamo y pino.

Comparation between retention media values of poplar and pinus.

\begin{tabular}{|lllllll|}
\hline Especie & $\begin{array}{c}\text { Media de } \\
\text { tratamientos }\end{array}$ & $\begin{array}{c}\text { Media } \\
\text { control }\end{array}$ & $\begin{array}{c}\text { Diferencia } \\
\text { de medias }\end{array}$ & $>$ o $<$ & Test & $\begin{array}{c}\text { Aprueba/ } \\
\text { Rechaza }\end{array}$ \\
\hline I-214 & 5,98 & 7,77 & 1,78 & $<$ & 2,137 & Aprueba \\
I-488 & 6,31 & 7,77 & 1,46 & $<$ & 2,137 & Aprueba \\
I-63/51 & 5,31 & 7,77 & 2,46 & $>$ & 2,137 & Rechaza \\
\hline
\end{tabular}




\section{CUADRO 3}

Características de la madera procedente de polines impregnados con CCA.

Characteristics of wood coming from utility poles impregnated with CCA.

\begin{tabular}{|lccccc|}
\hline Especie & $\begin{array}{c}\left.\text { Retención (kg óx } / \mathrm{m}^{3}\right) \\
\text { Prom (Desv.Est) }\end{array}$ & $\begin{array}{c}\text { Rc } \\
(\mathrm{MPa})\end{array}$ & $\begin{array}{c}\text { MOEc } \\
(\mathrm{MPa})\end{array}$ & $\begin{array}{c}\text { MORf } \\
(\mathrm{MPa})\end{array}$ & $\begin{array}{c}\text { MOEf } \\
(\mathrm{MPa})\end{array}$ \\
\hline $\mathrm{I}-214$ & $6,14(0,51)$ & 43,29 & $3.957,3$ & 70,54 & $7.070,8$ \\
$\mathrm{I}-488$ & $6,01(0,23)$ & 38,52 & $3.296,8$ & 60,27 & $7.590,5$ \\
I-63/51 & $6,36(0,50)$ & 37,83 & $2.958,1$ & 64,34 & $6.384,4$ \\
Pino & $6,65(0,87)$ & 34,23 & $3.581,0$ & 59,80 & $5.737,2$ \\
\hline
\end{tabular}

Donde:

Rc: Tensión máxima o de ruptura en compresión paralela a las fibras

MOEc: Módulo de elasticidad en compresión paralela a las fibras

MORf: Módulo de ruptura en flexión estática

MOEf: Módulo de elasticidad en flexión estática

nificativas en el comportamiento de algunos híbridos en los distintos ensayos a que fueron sometidos y la dispersión de cada uno de los datos para cada especie.

Para la tensión máxima o de ruptura, se aprecian diferencias entre el pino y los tres híbridos de álamo. Contrario a lo que se podría haber pensado a priori, estas diferencias fueron a favor de los híbridos de álamo. La mayor diferencia, comprobada estadísticamente, se encontró entre la madera impregnada del híbrido I-214 y la madera impregnada de pino. En cuanto a los módulos de elasticidad determinados en compresión paralela se observó que existe diferencia estadísticamente significativa entre el híbrido I-214 y el pino. Los híbridos I-488 e I-63/51 no presentan diferencias estadísticamente significativas. Como el efecto carga es común en elementos utilizados como polines, resulta ser interesante el hecho de que las mejores propiedades se concentren en el híbrido I-214.

\section{CUADRO 4}

Síntesis del test de Dunnet aplicado a la tensión máxima (MPa) y módulo de elasticidad (MPa), determinados en compresión paralela a la fibra en madera impregnada de los distintos híbridos y de la especie control.

Synthesis of Dunnet Test applied to maximum tension (MPa) and modulus of elasticity (MPa), based on compression parallel to grain in impregnated wood of different hybrids and the control species.

\begin{tabular}{|llll|}
\hline Especies & I-214 & I-488 & I-63/51 \\
\hline Media de los híbridos en tensión máxima & 43,29 & 38,53 & 37,83 \\
Media control en tensión máxima & 34,23 & 34,23 & 34,23 \\
Diferencia de medias & 9,2 & 4,44 & 3,74 \\
Valor del test & 2,3 & 2,3 & 2,3 \\
Aprueba/Rechaza & Rechaza* & Rechaza* & Rechaza* \\
\hline Media de los híbridos en módulo de elasticidad & $3.957,3$ & $3.296,8$ & $2.958,1$ \\
Media control en módulo de elasticidad & 3.581 & 3.581 & 3.581 \\
Diferencia de medias & 397,766 & 262,705 & 238,365 \\
Valor del test & 349,6 & 349,6 & 349,6 \\
Aprueba/Rechaza & Rechaza* & Aprueba & Aprueba \\
\hline
\end{tabular}

* significancia a $95 \%$ de probabilidad con 16 grados de libertad. 


\section{CUADRO 5}

Síntesis del test de Dunnet aplicado al módulo de ruptura (MPa) y módulo de elasticidad $(\mathrm{MPa})$, determinados en flexión estática en madera impregnada de los distintos híbridos y de la especie control.

Synthesis of Dunnet Test applied to modulus of rupture (MPa) and modulus of elasticity (MPa), based on static bending in impregnated wood of different hybrids and the control species.

\begin{tabular}{|llll|}
\hline Especies & I-214 & I-488 & I-63/51 \\
\hline Media de los híbridos en módulo de ruptura & 70,54 & 60,27 & 64,34 \\
Media control en módulo de ruptura & 59,8 & 59,8 & 59,8 \\
Diferencia de medias & 10,74 & 0,47 & 3,56 \\
Valor del test & 8,24 & 8,24 & 8,24 \\
Aprueba/Rechaza & Rechaza* & Aprueba & Aprueba \\
& & & \\
\hline Media de los híbridos en módulo de elasticidad & $7.070,8$ & $7.590,5$ & $6.384,4$ \\
Media control en módulo de elasticidad & $5.737,2$ & $5.737,2$ & $5.737,2$ \\
Diferencia de medias & $1.333,6$ & $1.854,2$ & 647,2 \\
Valor del test & 1.529 & 1.529 & 1.529 \\
Aprueba/Rechaza & Aprueba & Rechaza* & Aprueba \\
\hline
\end{tabular}

* significancia a $95 \%$ de probabilidad con 16 grados de libertad.

Para el módulo de ruptura en flexión se aprecian valores mayores en el híbrido I-214, y se determinan los menores valores para madera de pino, al igual que en el módulo de elasticidad en compresión paralela. Para el caso del módulo de elasticidad determinado en flexión estática, se determinó que el híbrido I-488 presenta mayores valores con respecto a madera de pino.

Es importante señalar que, dependiendo del esfuerzo, son distintos los híbridos que presentan mayores valores de resistencia comparados con la especie control. Es así como los tres híbridos presentan un valor de tensión máxima mayor que el pino y que solamente el híbrido I-214 presenta un mayor módulo de elasticidad cuando la madera se somete a un ensayo de compresión paralela a las fibras. En el ensayo de flexión estática diferentes híbridos destacan en el esfuerzo, por lo que es importante determinarlos y seleccionar la opción que maximice la resistencia de cada uno de ellos.

De este análisis se deduce que es importante diferenciar las potencialidades de los diferentes híbridos dependiendo del esfuerzo al que éstos se van a someter o a la especie que se desea sustituir.

\section{CONCLUSIONES}

La impregnación de los polines de distintos híbridos de álamo presentó buenos resultados, por cuanto al someterlos al mismo proceso de impregnación que polines de pino, se lograron buenos resultados en los híbridos I-214 e I-488. Para el híbrido I-63/51 es necesario hacer algunas variaciones al proceso.

Las propiedades mecánicas de la madera proveniente de los polines impregnados de los distintos híbridos permitieron evidenciar un posible uso de la madera de álamo, pues éstos presentaron resistencia mayor a la madera proveniente de polines impregnados de pino. En este sentido destaca entre estos tres híbridos el I-214, por tener los valores más altos en compresión paralela y módulo de ruptura en flexión, principales esfuerzos a los cuales se someten estos productos. 


\section{BIBLIOGRAFIA}

(1) FRAGA, A., F. TAPIA. Mejoramiento de la rentabilidad de los cultivos asociados a los álamos. Chillán. INIA. 2001. 13 p.

(2) INSTITUTO FORESTAL (INFOR). Mercado para trozas de postes y polines. Homepage 2002 [citado 1 de octubre de 2004]. Disponible en

http://www.infor.cl/webinfor/pwsistemagestion/mg 03 mercado/posypol/merpyp.htm.

(3) SANHUEZA, A., BOURKE, M., CHACON, I., PEREZ, C., ALVAREZ DE ARAYA, M., SANCHEZ, G., PINTO, C. Cultivo del Álamo (Populus spp). Parte 2. CONAF. Santiago. Chile. 1998. 214 p.

(4) SANHUEZA, A. El Álamo. Revista el Campesino 1996, $\mathrm{N}^{\circ} 4$ p. $4-10$.

(5) SANHUEZA, A. Cultivo del álamo. Santiago. CONAF. 2000. 214 p.

(6) INSTITUTO NACIONAL DE NORMALIZACION (INN) - Chile. Clasificación de maderas comerciales según su durabilidad natural. 1987, NCh 789 Of. 87.

(7) DIAZ-VAZ, J. Alamo. Revista Lignum. 1991. № 5. p. 24.

(8) PUETTMANN, M., SCHIMDT, E. Boron diffusion treatment of aspen lumber stored under various relative humidities. Forest Products Journal, 1997, vol 47, $\mathrm{N}^{\circ} 10$, pp. 47-50.

(9) Lignum, Durabilidad es el principal argumento: Madera impregnada es más barata que la natural. Página de la construcción. 2000. p. 43-44.

(10) CUEVAS, H. 2002. Propiedades mecánicas de Populus $x$ euramericana (Dode) Guinier creciendo en Valdivia, Chile. Revista Bosque, 2002, vol. 23, No 2, pp. 93-98.

(11) IBARRA, I. Impregnación de madera aserrada de populus $\mathrm{x}$ euramericana cv I-488 con CCB utilizando el método Bethell. Tesis de Ingeniería Forestal. Facultad de Ciencias Forestales. Universidad de Talca, 2003. 52 p.

(12) VERDUGO, J. Evaluación del comportamiento frente a la impregnación de dos variedades de álamo presentes en la Séptima Región. Tesis de Ingeniería Forestal. Facultad de Ciencias Forestales, Universidad de Talca. 2003. 77 p.

(13) PEREDO, F. Factibilidad técnica del uso de polines impregnados de álamo. Tesis de Ingeniería en Industria de la Madera. Facultad de Ciencias Forestales. Universidad de Talca, 2003, 96 p.

(14) INSTITUTO NACIONAL DE NORMALIZACION (INN) Madera - Determinación de humedad. 1984. NCh 176/1 Of. 84.
(15) Madera - Determinación de densidad, 1986, NCh $176 / 2$ Of. 86.

(16) _ Madera preservada - Clasificación y requisitos, 1996, NCh 819, Of. 96.

(17) ___ Madera - Selección, obtención y acondicionamiento de muestras y probetas para la determinación de las propiedades físicas y mecánicas, 1986, NCh 968, Of. 86.

(18) _____ Madera - Determinación de las propiedades mecánicas - Ensayo de compresión paralela, 1985, NCh 973 , Of. 85.

(19) ___ Madera - Determinación de las propiedades mecánicas - Ensayo de flexión estática. 1985. NCh 987 Of. 85.

(20) PEREZ, V. Manual de propiedades físicas y mecánicas de las maderas que crecen en Chile. INFOR, 1983, 398 p.

(21) PEREZ V. Manual de construcción en maderas. $2^{\mathrm{a}}$ ed. Instituto Forestal. Santiago. Chile, 1991 vol. 1. N ${ }^{\circ} 10$, $340 \mathrm{p}$.

(22) BURDON, R., HARRIS, J. Wood density in radiata pine clones on four different site. New Zealand Journal of Forestry Science. 1973, vol. 3, pp. 286-303.

(23) HARRIS, J. Wood quality of radiata pine. Appita, 1981, vol. 35, pp. 211-215

(24) BAMBER, R., BURLEY, J. The wood properties of radiata pine. Commonwealth Agriculture Bureau, London. 1983. 84 p.

(25) WILKES, J. Variation in wood density of Pinus radiata in New South Wales, Australia. Canadian Journal of Forest Research. 1988, vol. 19, pp. 289-294.

(26) RINGO, W., KLEM, G. Effect of ring with on density and tracheid length in the wood of Pinus radiata. Ind. $J$. For, 1989, vol. 12, pp. 179-182.

(27) ZOBEL, B., VAN BUIJTENEN, J. Wood variation: Its causes and control. Springer-Verlag, Berling. 1989. 363 p.

(28) COWN, D. McCONCHIE, D. YOUNG, G. Radiata pinewood properties survey. FRI bull $\mathrm{N}^{\circ} 50$, Rotorua. New Zealand. 1991. 50 p.

(29) VELIZ, G. Determinación de propiedades físicas y mecánicas en madera de híbridos de populus (I-488 e I-63/ 51). Tesis de Ingeniería Forestal. Facultad de Ciencias Forestales. Universidad de Talca, 2003, 119 p.

(30) SEPULVEDA, S. Medición de la dureza de la madera en árboles en pie en cinco híbridos de álamo mediante un método no destructivo, el Pilodyn. Tesis de Ingeniería Forestal. Facultad de Ciencias Forestales. Universidad de Talca, 2004, 54 p.

(31) F.A.O. Los chopos en la producción de madera y utilización de tierras. Italia, 1957, 525 p. 